\title{
FAK-targeting PROTAC as a chemical tool for the investigation of non-enzymatic FAK function in mice
}

Dear Editor,

Animal models, most commonly mice, that lack a protein of interest play an important role in phenotypic and functional studies of a target gene, allowing researchers to answer various biological questions (Chaible et al., 2010). At present, a variety of tools act at the DNA or RNA level to enable researchers to model gene function (and thus protein) deficiency, including nucleic acid-based RNA interference (Elbashir et al., 2001), antisense oligonucleotides (Schoch and Miller, 2017), and genome editing-based CRISPR-Cas9 (Doudna and Charpentier, 2014) strategies. However, challenges remain. RNA and DNA-based technologies lack exquisite temporal control of the target gene at specified time points in an organism's development, and they fail to realize acute and reversible target gene function (Chan, 2013). These shortcomings have garnered widespread concern in both fundamental research and drug development. Furthermore, gene knockout will often lead to embryonic lethality, precluding the study of post-embryonic pathophysiological functions of target genes and proteins of interest (Dhanjal et al., 2017).

Proteolysis targeting chimera (PROTAC) is a novel chemical knockdown technology for the post-translational study of proteins of interest. PROTACs are hetero-bifunctional small molecules, which can drive E3 ubiquitin ligase to bind with the target protein, resulting in ubiquitination of the target protein and consequent proteasome-mediated degradation (Raina and Crews, 2010) (Fig. 1A). Unlike classic inhibitors, PROTAC eliminates rather than inhibits both enzymatic and non-enzymatic protein functions. Furthermore, unlike nucleic acid (e.g., siRNA) and genome editing-based (e.g., CRISPR-Cas9) strategies (Cong et al., 2013; Deng et al., 2014), the small molecule-based PROTAC approach is capable of degrading target proteins without requiring any genetic manipulation, guaranteeing the integrity and stability of the genome, which especially suitable for knockdown of embryonic lethal protein. Thus, PROTACs offer significantly broader therapeutic applicability than DNA or RNA-targeting strategies for protein knockdown in vivo.
Focal adhesion kinase (FAK), an embryonic lethal protein, exerts kinase-dependent enzymatic functions and kinaseindependent scaffolding functions (Hall et al., 2011). Both functions are crucial in reproduction and early embryonic development (Gungor-Ordueri et al., 2014). Many essential non-enzymatic functions of FAK cannot be investigated with reported FAK kinase inhibitors. To the best of our knowledge, the PROTAC strategy has not been used to study the nonenzymatic function of FAK in vivo. It is also unknown whether FAK PROTACs will yield different phenotypes or reveal different FAK functions than kinase-dependent FAK inhibitors in vivo. For these reasons, we have chosen FAK as a target to demonstrate the potential utility of the PROTAC strategy for the study of non-enzymatic protein function in mouse reproductive system in vivo.

Based on the previous studies of our laboratory, we synthesised the FAK-targeting PROTAC library with FAK ligand of PF562271, cereblon (CRBN)-based E3 ubiquitin ligase ligand of thalidomide, and a variable length of polyethylene glycol or alkyl linkers (Fig. S1). Firstly, we screened the degradation effect of FAK targeting PROTAC molecules in mice primary reproductive related cells. We separated and purified testis-related cells, including primary Sertoli cells and primary germ cells, from $6 \mathrm{dpp}$ C57BL/6N mice, and tested whether degradation resulted from FAK PROTAC library molecules in these primary cells. A remarkable degradation effect, with a $\mathrm{DC}_{50}$ of $1.3 \mathrm{nmol} / \mathrm{L}$ for primary Sertoli cells and $0.4 \mathrm{nmol} / \mathrm{L}$ for primary germ cells, was observed from FAK-PROTAC library molecules, which we confirmed it is FC-11, a PROTAC from our previous reported work (Gao et al., 2019) (Fig. 1B-D). The optimized synthesis route of FC-11 was shown in Supplementary Materials (Scheme 1).

Next, in order to overcome the defect of FAK knockdown in vivo caused by existing genetic tools and to clarify the effect of PROTAC tools on the non-enzymatic function of protein in the mouse reproductive system, a few critical issues need to be addressed: 1. Can FC-11 degrade FAK in vivo? 2. If it can, is there any different phenotypes between FAK PROTAC and FAK inhibitor? 3. Is the FAK protein degradation reversible? 
A

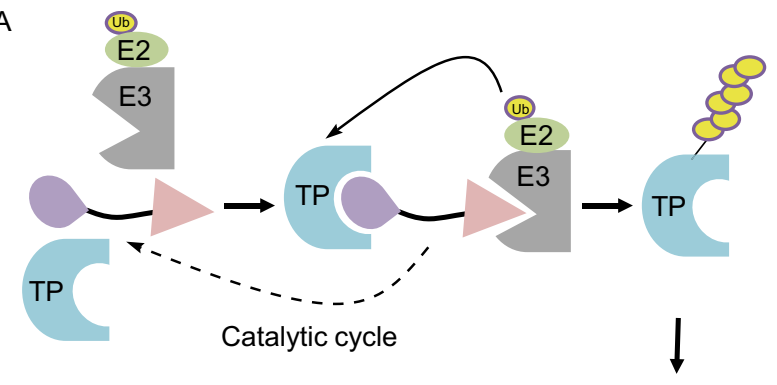

Degrade the entire target protein

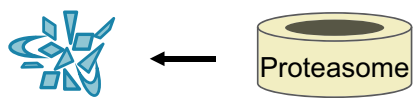

FAK PROTAC

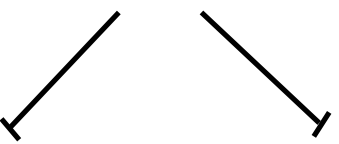

Enzymatic function

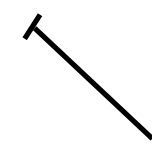

Non-enzymatic function

FAK inhibitor

C

Primary sertoli cells ( $\mathrm{nmol} / \mathrm{L})$

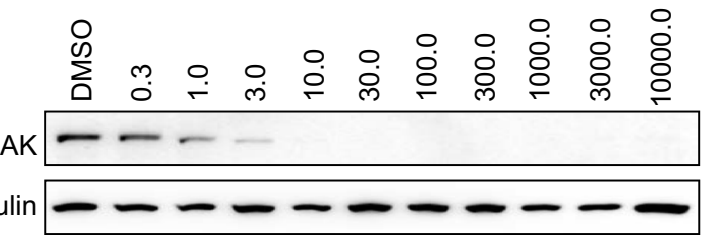

$\beta$-Tubulin $---\infty-\infty-\infty$

E

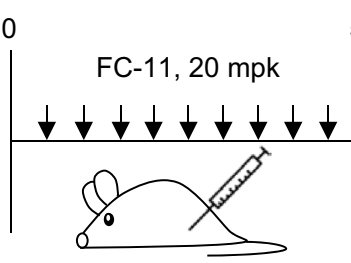

F
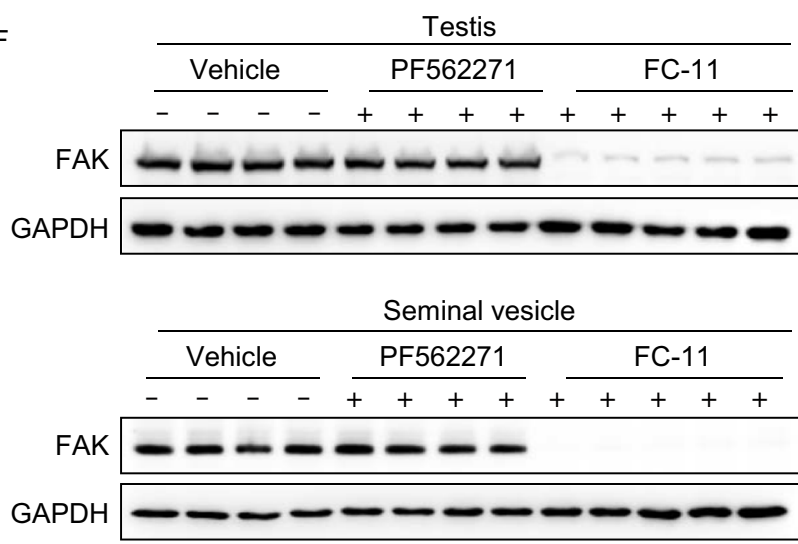

B

FAK Ligand: PF562271 - Linker - CRBN Ligand: Pomalidomide<smiles>CNc1ncc(C(F)(F)F)c(NCc2cccnc2N(C)S(C)(=O)=O)n1</smiles><smiles>NCCOCCOCCn1cc(C(=O)Nc2ccccc2)nn1</smiles>
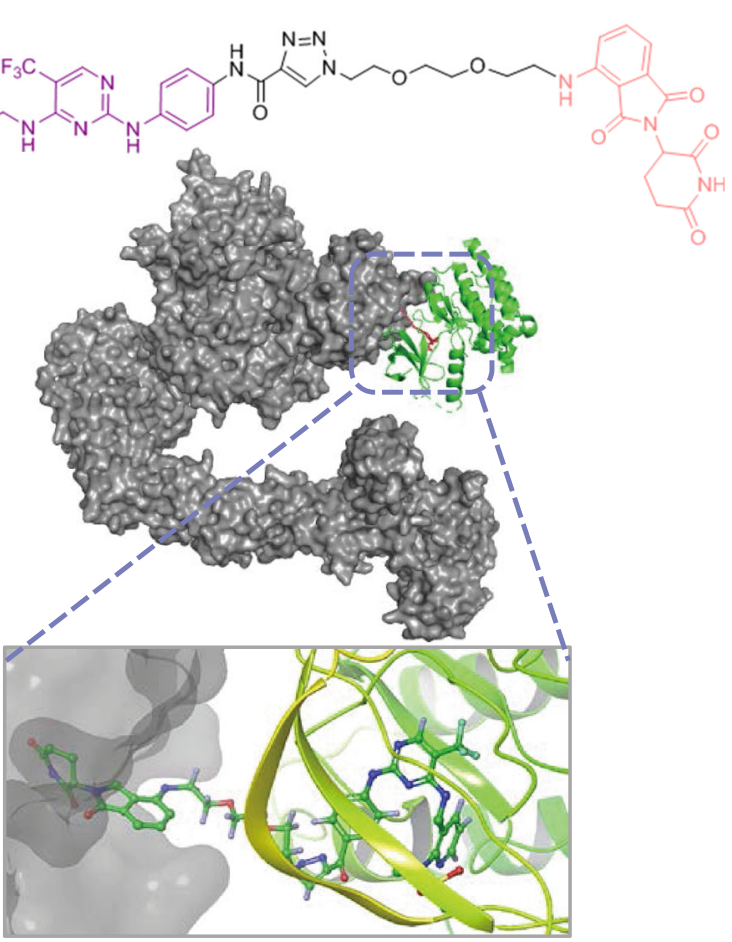

D

Primary germ cells (nmol/L)

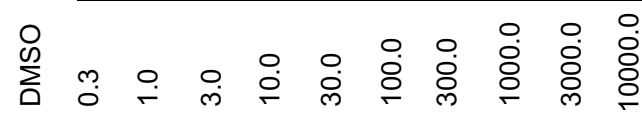

$\beta$-Tubulin

FAK
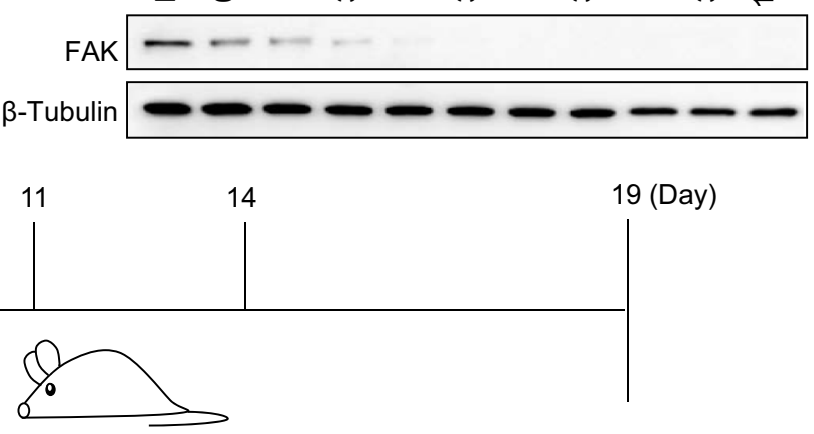

19 (Day)
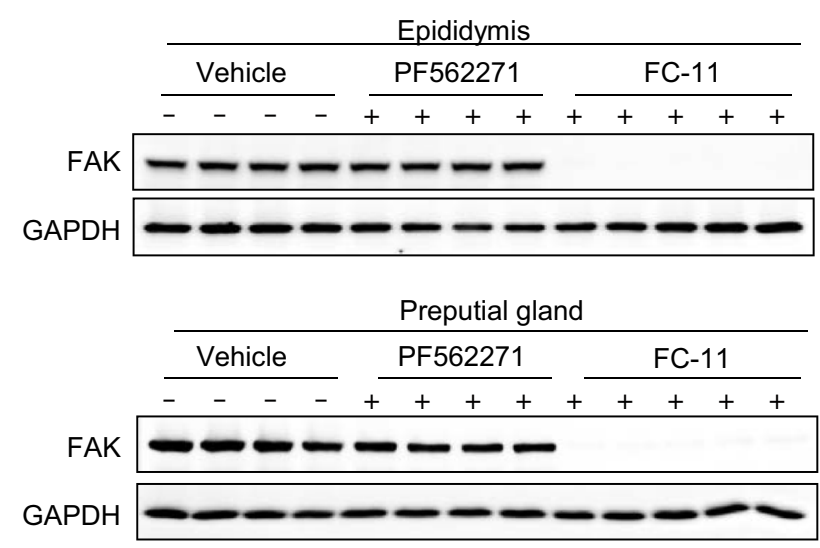
Figure 1. Rapid and reversible FAK knockdown by FAK targeting PROTAC in male mice reproductive organs. (A) Schematic depiction of the PROTAC strategy. FAKPROTAC tool can act on both enzymatic and non-enzymatic functions of FAK, while FAK inhibitor only act on the enzymatic function of FAK. TP, Target Protein. (B) Chemical structure of FAK PROTAC, as shown in the upper portion. Binding mode of PROTAC (ball stick), FAK (PDB 5TOB, green) and CRL4-CRBN (PDB 2HYE and 4Cl3, gray) was simulated by Pymol. (C and D) FAK protein degradation in mice primary Sertoli cells and primary Germ cells, the cells were treated at the indicated doses of FC-11 for $8 \mathrm{~h}$. (E) Schematic depiction of FC-11 treated mice for FAK degradation and recovery. (F) FC-11 leads to more extensive FAK degradation in testis, epididymis, seminal vesicle and preputial gland, respectively. Each lane represented a single mouse ( $n=4$ or 5 ). (G) The recovery ability of FAK in testis, epididymis, seminal vesicle and preputial gland in the indicated days after withdraw administration $(n=6)$. All western blots are the representatives from at least 3 experiments.

Encouraged by the results from primary cells, we continued to test FC-11 induced FAK degradation in the reproductive tissues (testis, epididymis, seminal vesicle and preputial gland) of male mice in vivo (Fig. S2). Ten-week-old male $\mathrm{C} 57 \mathrm{BL} / 6 \mathrm{~N}$ mice were administered intraperitoneal injections of FC-11 (20 mg/kg, twice daily [BID]), PF562271 (10 $\mathrm{mg} / \mathrm{kg}, \mathrm{BID})$, or vehicle control over a 5 day period (Fig. 1E). After 5 days treatment, all FC-11 treated mice exhibited a more than $90 \%$ reduction of FAK and phosphor FAK $^{\text {tyr397 }}$ in the tested reproductive tissues, while PF562271 had no effect on the level of FAK protein, but had an inhibitory effect on the phosphor FAK ${ }^{\text {tyr397 }}$ levels (Figs. 1F and S3). These results demonstrated that $\mathrm{FC}-11$ can rapidly and efficiently degrade FAK in the reproductive tissues of male mice. In addition, the location and expression of FAK in the testis were detected by immunofluorescent. Immunostaining revealed that FAK was mainly localized to the basal compartment of seminiferous tubules, which was consistent with previously published data (Siu et al., 2009) (Fig. S4). As above, FC-11 treatment significantly decreased the cytoplasmic expression of FAK, while PF562271 treatment had no effect on FAK levels, again demonstrating the totally different mechanisms of action of the FAK-PROTAC protein degrader FC-11 and the FAK inhibitor PF562271.

Whether FAK protein could be recovered to normal levels after withdrawal of treatment? The mice were raised normally for 2, 4, 6, 9, and 14 days, after withdrawing drug treatment (Fig. 1E). FAK levels recovered gradually over time after FC-11 withdrawal. Except in the preputial gland, the level of FAK in mouse reproductive organs (testis, epididymis and seminal vesicle) was almost normal at 14 days after FC-11 withdrawal. FAK levels in the preputial gland only recovered about $40 \%$ in 14 days (Fig. 1G). The above results indicate that $\mathrm{FC}-11$ can achieve reversible regulation of FAK in mice.

Based on the above results, FAK-PROTAC showed a potent and reversible FAK degradation in mouse reproductive tissues of male mice, which indicated that FC-11 can be used as a biological tool for FAK knockdown in vivo. Next, the possible functional consequence differences between FAK PROTAC and FAK inhibitor in mouse reproductive system of male mice were further observed. We administered FC-11 (20 mg/kg, BID), PF562271 (10 mg/kg, BID), and vehicle control to 10 week old male C57BL/6N mice via intraperitoneal injections for 13 days. After 13 days treatment, all FC-11 treated males exhibited a significant reduction in the weight or size of the testis, epididymis, seminal vesicle, and preputial gland of $24.2 \%, 37.5 \%, 51.6 \%$ and $90 \%$ of vehicle control, respectively. No reduction was observed in PF562271-treated mice compared to the vehicle-treated group (Figs. 2A and S5). In addition, the number of viable sperm from the caudal epididymis was markedly decreased in FC-11 treated mice. Analysis of sperm motility in FC-11 treated males also revealed a significant (more than

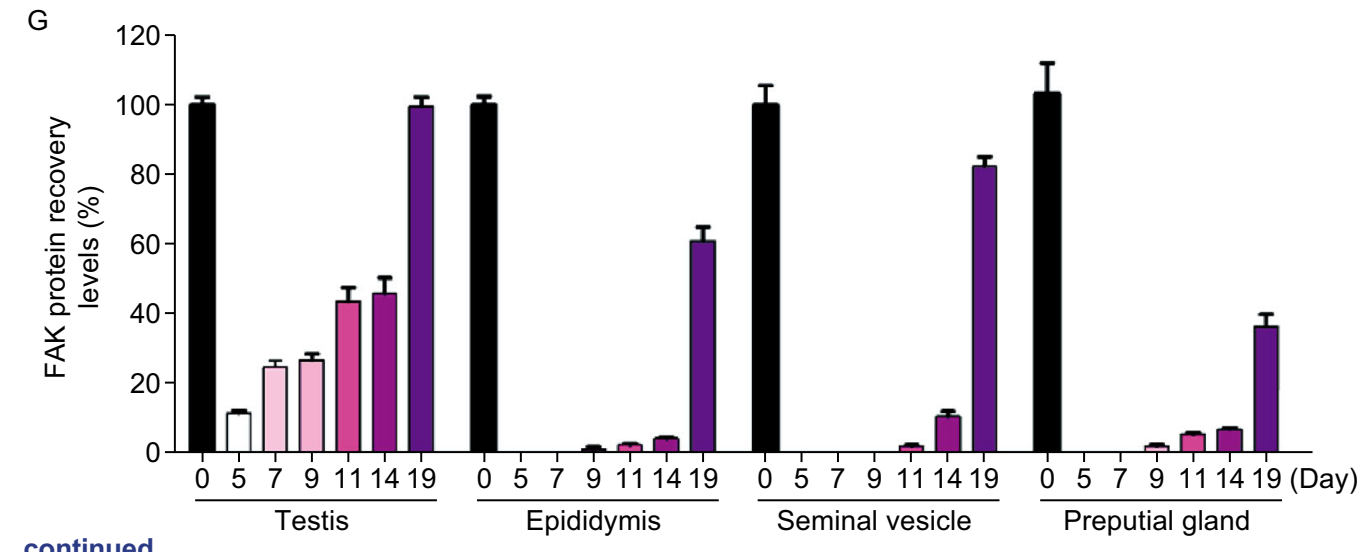

Figure 1. continued. 
A

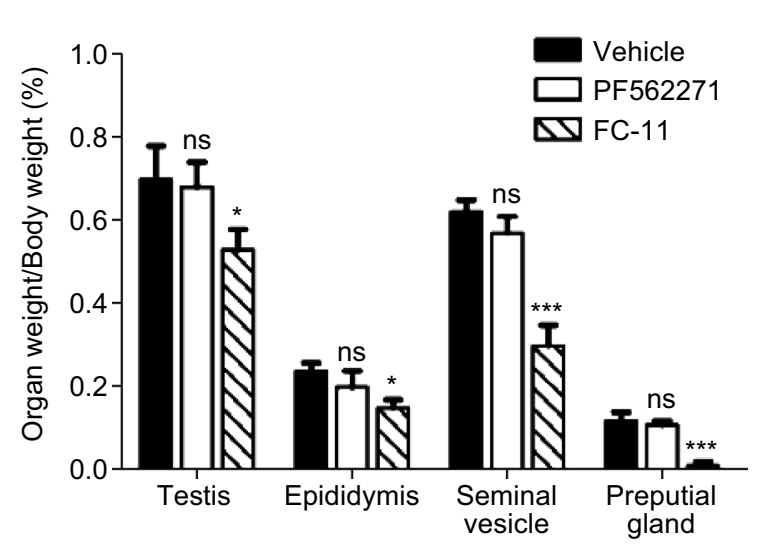

D
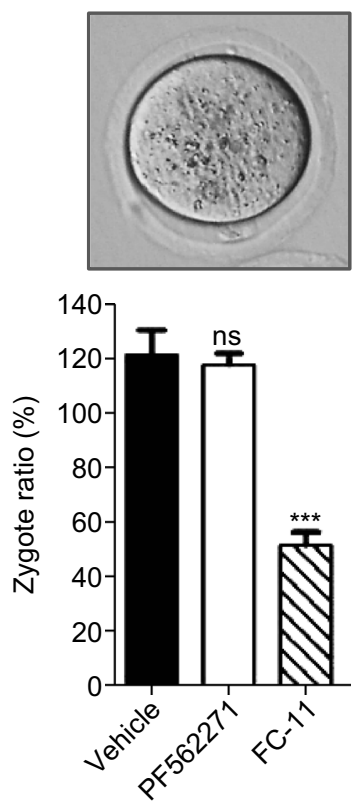

$\mathrm{H}$
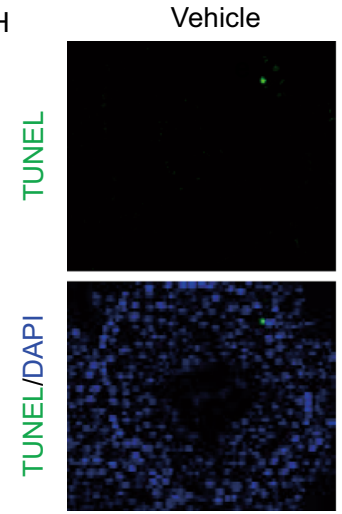

E
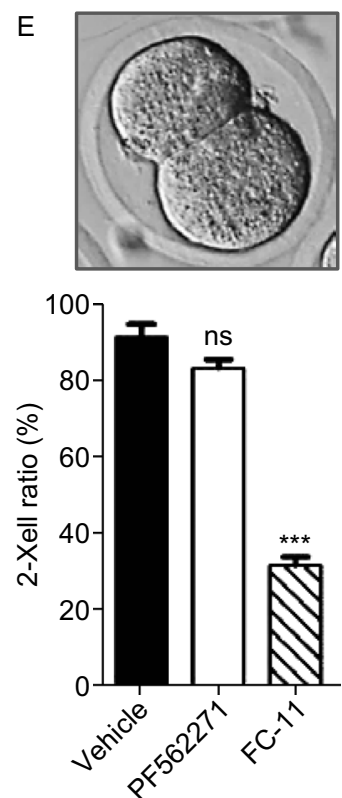

PF562271
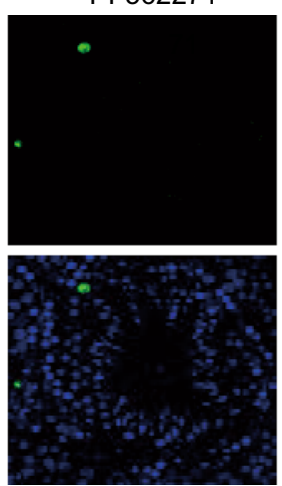
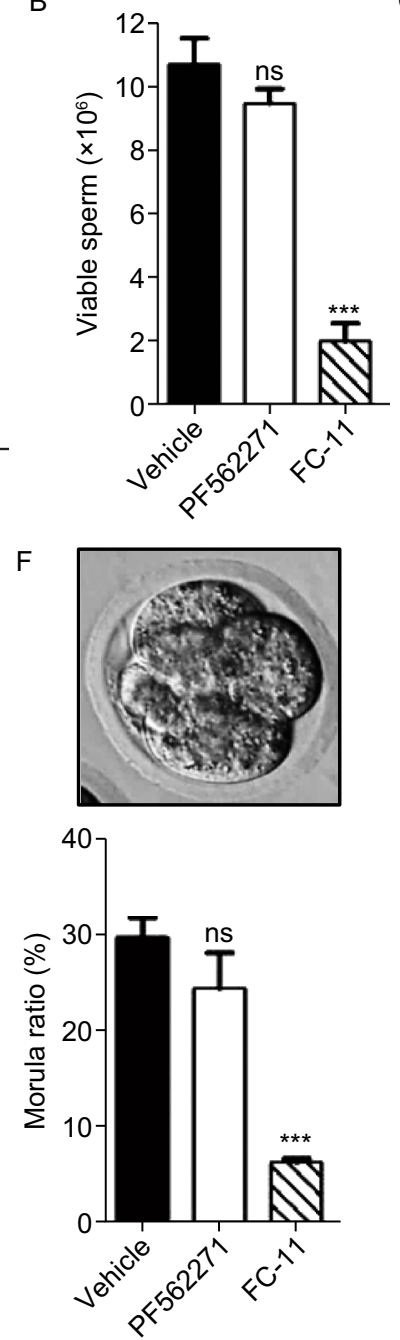

C

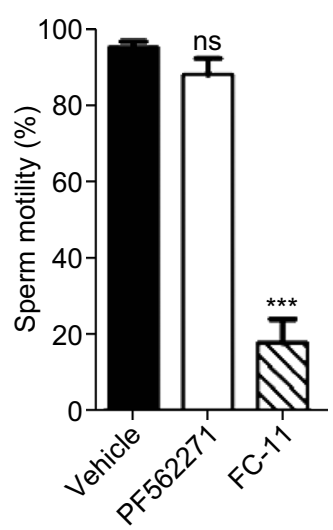

G
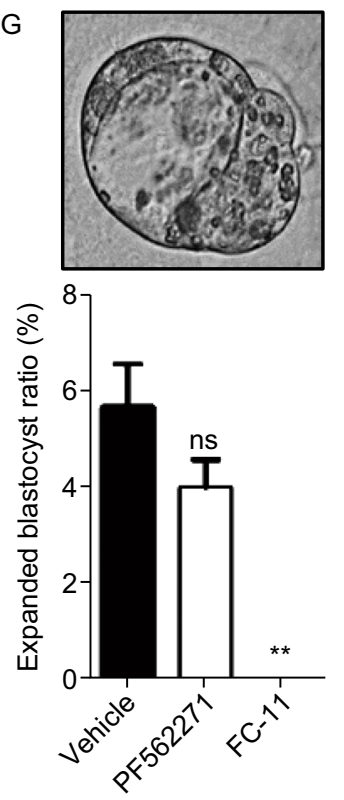

FC-11
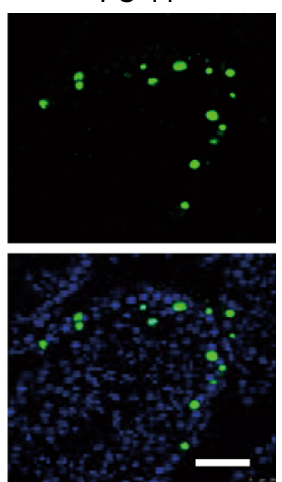

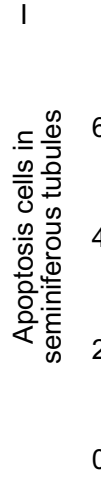

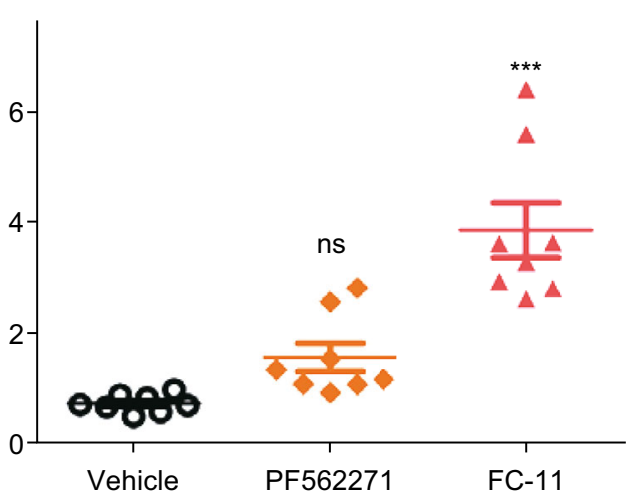


Figure 2. FAK PROTAC and FAK inhibitor showed different effects on reproductive tissues and sperm phenotypes in male mice. (A) Organ index of each group mice (Organ index $\%=$ organ weight/body weight $\times 100, n=6)$. (B and C) Characteristics of sperm phenotype (viable sperms and sperm motility) from each group $(n=6)$. (D-G) Statistical analysis of in vitro fertilization rate of spermatozoa and the development of pre-implantation mouse embryos in each group mice, the image represents the morphology of pre-implantation mouse embryos in various developmental stages $(n=6)$. $(\mathrm{H})$ TUNEL staining of testis sections from each group mice (bar, $50 \mu \mathrm{m}$ ). (I) Statistic data of apoptosis cells in seminiferous tubules of testis by TUNEL staining, about 160 seminiferous tubules are selected for statistic in each group. The graphs depict mean \pm SD of six mice, ns: no significant, ${ }^{\star} P<0.05,{ }^{* \star} P<0.01,{ }^{* \star *} P<0.001$ vs. vehicle group.

five fold) reduction compared to vehicle males. However, there was no significant decrease in sperm viability in PF562271 treated mice compared to vehicle control mice (Fig. 2B and 2C).

In addition, the fertilization rate and the development of 2-cell, morula, and expanded blastocyst embryos from FC11 treated mouse sperm was strongly impaired compared to embryos fertilized by sperm from vehicle mice, while the fertilization rate and embryos fertilized by sperm from PF562271-treated mice displayed normal or only slightly impaired embryonic development in vitro (Figs. 2D-G and S6). The observed decrease in sperm fertility and impaired embryo development in FC-11 treated males imply that FC11 has the potential to modulate the fertility of male mice. Furthermore, histopathology and apoptosis analysis of the seminiferous tubules of the testis showed that FC-11, but not PF562271, induced a significant increase in apoptosis of germ cells close to the base membrane of seminiferous tubules compared to vehicle (Figs. $2 \mathrm{H}, 2 \mathrm{I}$ and S7).

In summary, we have described developing a FAK-targeting PROTAC probe for chemical biology study of related non-enzymatic function of FAK in murine reproductive system. This novel strategy has an advantage over current FAK small molecule inhibitors because inhibitors are only applicable to the study of enzymatic functions, not the study of both enzymatic and non-enzymatic function. In this study, the result showed that FAK can be degraded by more than 90\% after representative degarder FC-11 treatment, and that it can be recovered to normal levels within two weeks after withdrawing treatment in vivo. In contrast to FAK inhibitor PF562271-treated mice, which exhibit an intact reproductive system, FC-11-treated FAK knockdown mice exhibit low sperm viability and motility, and subsequent decreased fertility and poor embryo development due to the impairment of non-enzymatic FAK functions.
Furthermore, in order to make better use of the FAK PROTAC tool in mice, we also detected the general distribution of FC-11 in mice with $20 \mathrm{mg} / \mathrm{kg}$ of FC-11 (BID) in 10 week old male $\mathrm{C} 57 \mathrm{BL} / 6 \mathrm{~N}$ mice through intraperitoneal injections for 5 days. The result displayed that FC-11 can not penetrate the blood-brain barrier, and we did not detect the FAK knockdown in brain of mice. However, FC-11 can work in other mice organs such as liver, spleen, lung, and kindey with different degradation degree, which may further broaden the potential application of FC-11 in other biological studies. In addition, from the celluar FAK selectivity analysis, we also found that FC-11 did not degrade the off-targets of PF562271 such as CDK1, CDK2, CDK7 and FLT3 (Roberts et al., 2008), but FC-11 showed a very slight protein degradation in proline-rich tyrosine kinase 2 (Pyk2) in SRD15 cell line, the homolog of FAK, which have a highly consistent in structure with FAK (45\% homology with FAK in amino acid sequence and $61 \%$ homology in catalytic domain) (Fig. S8) (Zheng et al., 1998).

Overall, our findings indicate that PROTACs can be used as chemical knockdown tools to study the non-enzymatic functions of proteins, shedding the constraints of traditional small molecule inhibitors. Unlike DNA- or RNA-based protein knockout technology, the PROTAC strategy knocks down target proteins directly, rather than acting on the genome or nucleic acid level, and is suitable for the functional study of embryonic-lethal proteins in adult organisms. Finally, PROTAC probes also provide exquisite temporal control, allowing the knockdown of a target protein of interest at specific developmental time points and enabling the recovery of the target protein after withdrawal of drug treatment.

\section{FOOTNOTES}

We thank Dr. Wei Wu and Dr. Qiuye Zhao from Tsinghua University for their kind help. We thank the laboratory animal research centre, Tsinghua University for the animal care and the in vitro fertility service support. This work was supported by the National Natural Science Foundation of China (Grant Nos. 81573277, 81622042, 81773567, and 81811530340), National Major Scientific and Technological Special Project for "Significant New Drugs Development" (Grant Nos. SQ2017ZX095003 and 2018ZX09711001).

Hongying Gao, Chunwei Zheng, Jian Du, Yue Wu, Yonghui Sun, Chunsheng Han, Kehkooi Kee and Yu Rao declare that they have no conflict of interest.

All institutional and national guidelines for the care and use of laboratory animals were followed.

\section{Hongying $\mathrm{Gao}^{1,2}$ (1) , Chunwei Zheng ${ }^{3}$ (D), Jian $\mathrm{Du}^{4}$,} Yue $\mathrm{Wu}^{1}$, Yonghui Sun ${ }^{1}$, Chunsheng $\operatorname{Han}^{3,5} \bowtie$ (i), Kehkooi Kee ${ }^{4 凶}$ (i) Yu Rao ${ }^{1 凶}$ (i)

${ }^{1}$ MOE Key Laboratory of Protein Sciences, School of Pharmaceutical Sciences, MOE Key Laboratory of Bioorganic Phosphorus Chemistry Chemical Biology, Tsinghua University, Beijing 100084, China 
2 Tsinghua University-Peking University Joint Center for Life Sciences, Beijing 100084, China

${ }^{3}$ State Key Laboratory of Stem Cell and Reproductive Biology, Institute of Zoology, Chinese Academy of Sciences, Beijing 100084, China

${ }^{4}$ Center for Stem Cell Biology and Regenerative Medicine, Department of Basic Medical Sciences, School of Medicine, Tsinghua University, Beijing 100084, China

${ }^{5}$ University of Chinese Academy of Sciences, Savaid Medical School, Beijing 100049, China

$\square$ Correspondence: hancs@ioz.ac.cn (C. Han), kkee@tsinghua. edu.cn (K. Kee), yrao@tsinghua.edu.cn (Y. Rao)

\section{OPEN ACCESS}

This article is licensed under a Creative Commons Attribution 4.0 International License, which permits use, sharing, adaptation, distribution and reproduction in any medium or format, as long as you give appropriate credit to the original author(s) and the source, provide a link to the Creative Commons licence, and indicate if changes were made. The images or other third party material in this article are included in the article's Creative Commons licence, unless indicated otherwise in a credit line to the material. If material is not included in the article's Creative Commons licence and your intended use is not permitted by statutory regulation or exceeds the permitted use, you will need to obtain permission directly from the copyright holder. To view a copy of this licence, visit http:// creativecommons.org/licenses/by/4.0/.

\section{REFERENCES}

Chaible LM, Corat MA, Abdelhay E, Dagli ML (2010) Genetically modified animals for use in research and biotechnology. Genet Mol Res 9:1469-1482

Chan AW (2013) Progress and prospects for genetic modification of nonhuman primate models in biomedical research. ILAR J 54:211-223

Cong L, Ran FA, Cox D, Lin S, Barretto R, Habib N, Hsu PD, Wu X, Jiang W, Marraffini LA et al (2013) Multiplex genome engineering using CRISPR/Cas systems. Science 339:819-823
Deng Y, Wang CC, Choy KW, Du Q, Chen J, Wang Q, Li L, Chung TK, Tang T (2014) Therapeutic potentials of gene silencing by RNA interference: principles, challenges, and new strategies. Gene 538:217-227

Dhanjal JK, Radhakrishnan N, Sundar D (2017) Identifying synthetic lethal targets using CRISPR/Cas9 system. Methods 131:66-73

Doudna JA, Charpentier E (2014) Genome editing. The new frontier of genome engineering with CRISPR-Cas9. Science 346:1258096

Elbashir SM, Harborth J, Lendeckel W, Yalcin A, Weber K, Tuschl T (2001) Duplexes of 21-nucleotide RNAs mediate RNA interference in cultured mammalian cells. Nature 411:494-498

Gao H, Wu Y, Sun Y, Yang Y, Zhou G, Rao Y (2019) Design, synthesis, and evaluation of highly potent FAK-targeting PROTACs. ACS Med Chem Lett. https://doi.org/10.1021/acsmedchemlett.1029b00372

Gungor-Ordueri NE, Mruk DD, Wan HT, Wong EW, Celik-Ozenci C, Lie PP, Cheng CY (2014) New insights into FAK function and regulation during spermatogenesis. Histol Histopathol 29:977989

Hall JE, Fu W, Schaller MD (2011) Focal adhesion kinase: exploring Fak structure to gain insight into function. Int Rev Cell Mol Biol 288:185-225

Raina K, Crews CM (2010) Chemical inducers of targeted protein degradation. J Biol Chem 285:11057-11060

Roberts WG, Ung E, Whalen P, Cooper B, Hulford C, Autry C, Richter D, Emerson E, Lin J, Kath J et al (2008) Antitumor activity and pharmacology of a selective focal adhesion kinase inhibitor, PF-562,271. Cancer Res 68:1935-1944

Schoch KM, Miller TM (2017) Antisense oligonucleotides: translation from mouse models to human neurodegenerative diseases. Neuron 94:1056-1070

Siu ER, Wong EW, Mruk DD, Porto CS, Cheng CY (2009) Focal adhesion kinase is a blood-testis barrier regulator. Proc Natl Acad Sci USA 106:9298-9303

Zheng C, Xing Z, Bian ZC, Guo C, Akbay A, Warner L, Guan JL (1998) Differential regulation of Pyk2 and focal adhesion kinase (FAK). The C-terminal domain of FAK confers response to cell adhesion. J Biol Chem 273:2384-2389
Hongying Gao and Chunwei Zheng have contributed equally to this work.

Electronic supplementary material The online version of this article (https://doi.org/10.1007/s13238-020-00732-8) contains supplementary material, which is available to authorized users. 